\title{
HIGH-PRECISION ASTROMETRIC MILLIMETER VERY LONG BASELINE INTERFEROMETRY USING A NEW METHOD FOR MULTI-FREQUENCY CALIBRATION
}

\author{
Richard Dodson $^{1}$, María J. Rioja ${ }^{1,2,3}$, Sol N. Molina ${ }^{4}$, and José L. Gómez ${ }^{4}$ \\ ${ }^{1}$ International Centre for Radio Astronomy Research, M468, The University of Western Australia, 35 Stirling Hwy, \\ Crawley, Western Australia 6009, Australia; richard.dodson@icrar.org \\ 2 CSIRO Astronomy and Space Science, 26 Dick Perry Avenue, Kensington WA 6151, Australia \\ ${ }^{3}$ Observatorio Astronómico Nacional (IGN), Alfonso XII, 3 y 5, E-28014 Madrid, Spain \\ ${ }^{4}$ Instituto de Astrofísica de Andalucía-CSIC, Glorieta de la Astronomía s/n, E-18008 Granada, Spain \\ Received 2016 August 10; revised 2016 November 22; accepted 2016 November 24; published 2017 January 12
}

\begin{abstract}
In this paper we describe a new approach for millimeter Very Long Baseline Interferometry (mm-VLBI) calibration that provides bona-fide astrometric alignment of the millimeter-wavelength images from a single source, for the measurement of frequency-dependent effects, such as "core-shifts" near the black hole of active galactic nucleus jets. We achieve our astrometric alignment by solving first for the ionospheric (dispersive) contributions using wide-band centimeter-wavelength observations. Second, we solve for the tropospheric (nondispersive) contributions by using fast frequency-switching at the target millimeter-wavelengths. These solutions can be scaled and transferred from low frequency to the high frequency. To complete the calibration chain an additional step is required to remove a residual constant phase offset on each antenna. The result is an astrometric calibration and the measurement of the core-shift between 22 and $43 \mathrm{GHz}$ for the jet in BL Lacertae to be $-8 \pm 5$, $20 \pm 6 \mu$ as, in R.A. and decl., respectively. By comparison to conventional phase referencing at centimeterwavelengths we are able to show that this core shift at millimeter-wavelengths is significantly less than what would be predicted by extrapolating the low-frequency result, which closely followed the predictions of the Blandford \& Königl conical jet model. As such it would be the first demonstration for the association of the VLBI core with a recollimation shock, normally hidden at low frequencies due to the optical depth, which could be responsible for the $\gamma$-ray production in blazar jets.
\end{abstract}

Key words: astrometry - quasars: individual (BL-Lac) - radio continuum: galaxies - techniques: interferometric

\section{INTRODUCTION}

Results of over eight years of monthly monitoring of a sample of blazars (the most luminous and variable BL Lac objects and flat-spectrum radio quasars) with the Very Long Baseline Array (VLBA) at $7 \mathrm{~mm}$ by the Boston University blazar group (Jorstad \& Marscher 2016) ${ }^{5}$ show that most $\gamma$-ray flares are simultaneous (within errors) with the appearance of a new superluminal component or a major outburst in the Very Long Baseline Interferometry (VLBI) core of the jet, defined as the bright, compact feature at its upstream end (see Marscher et al. 2008, 2010; Jorstad et al. 2013; Casadio et al. 2015a, 2015b). A burst in particle and magnetic energy density is therefore required when jet disturbances cross the radio core in order to produce $\gamma$-ray flares, which can naturally be explained by identifying the radio core with a recollimation shock (e.g., Daly \& Marscher 1988; Gómez et al. 1995, 1997; Marscher 2009, 2012; Mizuno et al. 2015; Martí et al. 2016).

On the other hand, the standard Blandford \& Königl conical jet model hypothesizes that the core is not a physical feature in the jet, but corresponds to the location at which the jet becomes optically thin, and therefore its position shifts with observing frequency (Blandford \& Königl 1979; Königl 1981; Lobanov 1998). This is conventionally referred to as the "coreshift". In this case the separation from the black hole is $r=r_{0} \nu^{\kappa}$, where $\nu$ is the frequency and $\kappa$ is a value close to -1 (Lobanov 1998; additionally there can be an offset from the nominal reference position). Multi-frequency VLBI

\footnotetext{
5 The VLBA-BU-BLAZAR monitoring program; see http://www.bu.edu/ blazars/VLBAproject.html.
}

observations at centimeter wavelengths have measured this core frequency shift in multiple sources, albeit without phasereferencing (e.g., Kovalev et al. 2008; O'Sullivan \& Gabuzda 2009; Sokolovsky et al. 2011; Fromm et al. 2015). Nevertheless phase-referenced VLBI observations have confirmed that the centimeter-wavelength radio core indeed is consistent with the optically thick-thin transition, in a smaller number of targets, such as 3C $395,4 \mathrm{C} 39.25,1038+528$, 3C 390.1, M 81, M 87, and 3C454.3 (Lara et al. 1994; Guirado et al. 1995; Rioja \& Porcas 1998; Ros et al. 2001; Hada et al. 2011; Martí-Vidal et al. 2011; Kutkin et al. 2014, respectively).

We have therefore two sets of results, one suggesting that the radio core corresponds to a recollimation shock while the other implies that it marks the transition between the optically thickthin jet regimes. A possible solution to reconcile these apparently contradicting observational results is to consider that the core is located parsecs away from the central black hole (e.g., Marscher et al. 2002; Chatterjee et al. 2011; Fromm et al. 2015) and consists of a recollimation shock that leads to $\gamma$-ray flares as new perturbations in the jet flow cross its position (e.g., Jorstad \& Marscher 2016). At this distance from the black hole the core is optically thin at millimeterwavelengths, while at longer wavelengths the core becomes optically thick, leading to the observed Blandford \& Königl core frequency shift.

We have performed numerical simulations to test this proposed model, using the finite-volume code Ratpenat, which solves the equations of relativistic hydrodynamics (Perucho et al. 2010, and references therein). The jet is launched with an initial over-pressure of 1.5 times that of the external medium in order to obtain a recollimation shock that can be identified with 

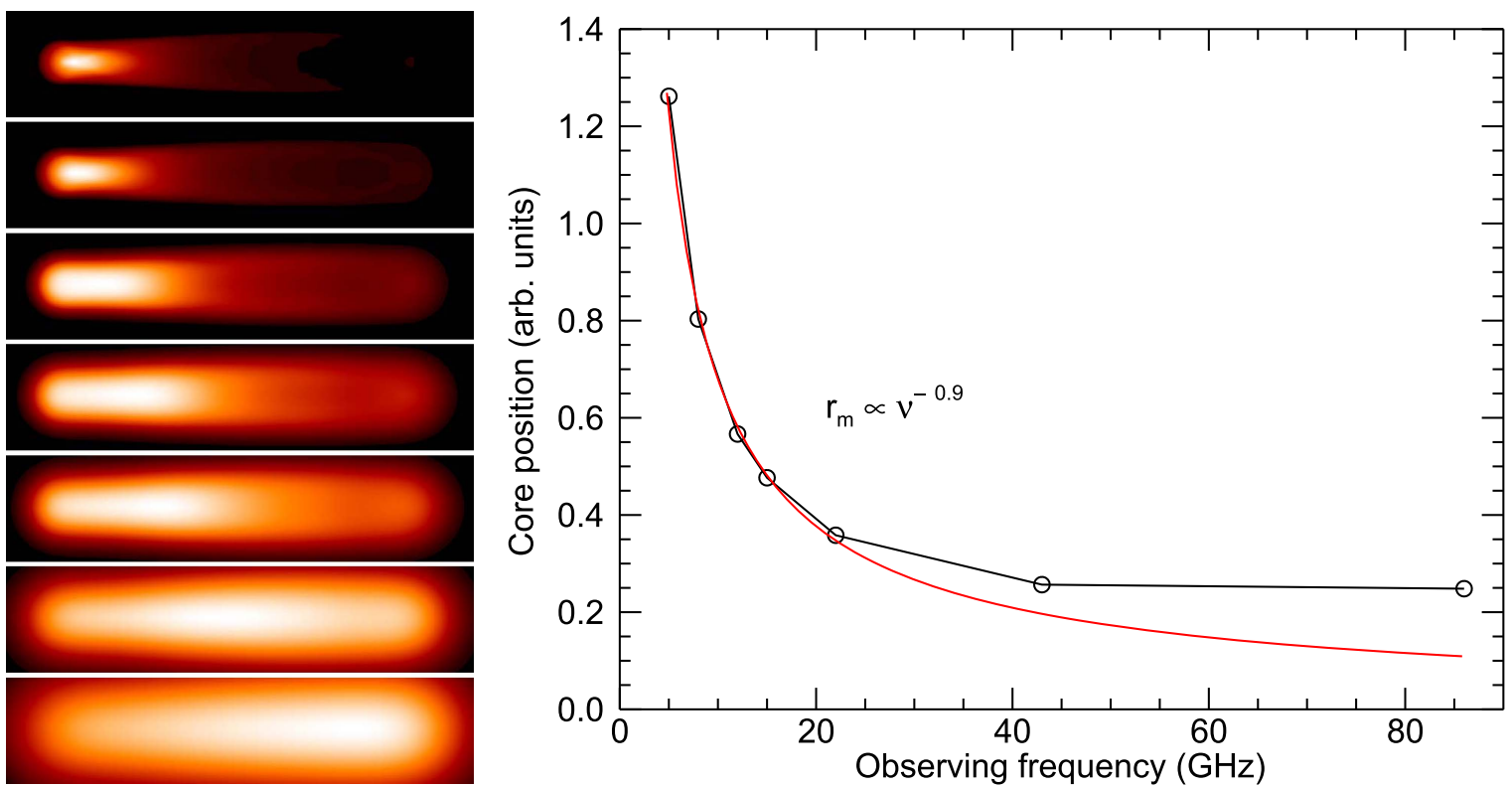

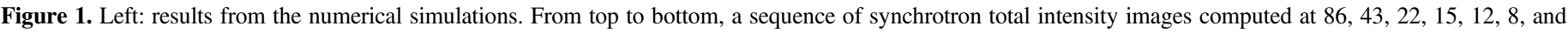

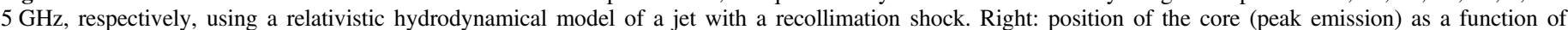

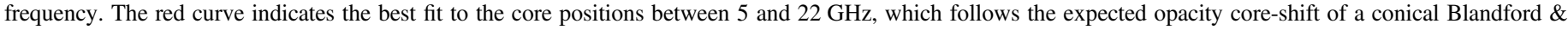

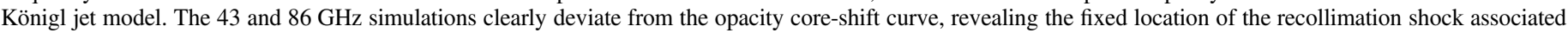
with the core.

the core. Using the hydrodynamical results as input, we have then computed the synchrotron emission at different observing frequencies (for details of the numerical model used see Gómez et al. 1995, 1997; Aloy et al. 2003). This is illustrated by Figure 1, which shows the sequence of total intensity images at the different frequencies, as well as the evolution of the core position with frequency. Full details of the simulations will be published elsewhere; here we just summarize the notunexpected conclusions, which are that, at centimeter-wavelengths $(5-22 \mathrm{GHz})$ the simulations reproduce the opacity coreshift of a Blandford \& Königl conical jet model, while at mmwavebands (43 and $86 \mathrm{GHz}$ ) the core position clearly departs from this behavior, revealing the recollimation shock at a fixed jet location.

Testing observationally whether the VLBI core at millimeter wavelengths is indeed consistent with the presence of a recollimation shock requires therefore bona-fide astrometric measurements of the core-shift spanning a wide range of centimeter and millimeter wavelengths. Confirmation of this model will support the hypothesis that the majority of the $\gamma$-ray flares in AGN jets are produced by the passing of new superluminal features through a pattern of recollimation shocks in the innermost jet regions, which would also include the VLBI core (Jorstad et al. 2013; Casadio et al. 2015b; Gómez et al. 2016). If our model is correct, we expect to see the Blandford \& Königl core-shift at cm-wavebands (5, 8.4, 15, and $22 \mathrm{GHz}$ ), with deviation from this behavior at mmwavebands ( 43 and $86 \mathrm{GHz}$ ). It should be also noted that at the innermost jet regions probed at mm-wavebands, other effects, such as radiative cooling or the parabolic jet shape found in M87 by Asada \& Nakamura (2012), may also lead to a departure from the opacity core-shifts of the conical Blandford \& Königl jet model. However none of these effects appears to affect the measurements performed by Hada et al. (2011), perhaps due to the progressive increase in the Doppler boosting as the jet accelerates in the innermost jet regions (Asada et al. 2014). Additionally, recent space VLBI RadioAstron observations of BL Lac at a record angular resolution of $21 \mu$ as have found evidence for the association of the radio core with a recollimation shock (Gómez et al. 2016), providing extra motivation for this work.

Conventional phase referencing (PR) (Alef 1988; Beasley \& Conway 1995) is the best approach for this analysis in centimeter-wavelength observations. The source/frequency phase referencing (SFPR) method works well for millimeterwavelength observations, and has been demonstrated with frequencies as high as $130 \mathrm{GHz}(2 \mathrm{~mm})$ (Rioja et al. 2015). However SFPR requires a second calibrator source with-in about $10^{\circ}$ of the target. The density of calibrators at $86 \mathrm{GHz}$, or even at $43 \mathrm{GHz}$, is not sufficient to guarantee that a suitable source will be within this range. Indeed, this was the case for the source discussed in this paper: BL Lac. Therefore we have developed a method built on the frequency phase transfer (FPT) approach that underpins SFPR, but does not require a second source. The description and validation of this method is the focus of this paper.

\section{OBSERVATIONS}

The observations presented here were carried out on 2013 July 5 with the VLBA targeting the jet in BL Lac, and are part of a series of similar experiments on a sample of blazar sources aimed to test the correspondence of the mm-VLBI core with a recollimation shock. The analysis here provides the demonstration and explanation of a new technique we have developed for single source astrometric $\lambda$-astrometry mm-VLBI, where the high-frequency images are astrometrically registered to a lower-frequency image.

All observations were made at $2 \mathrm{Gbps}$ with $32 \mathrm{MHz}$ intermediate frequency (IF) bands. Prime calibrators, 3C 345 and 3C 84, were observed for all frequency bands. Ten blocks of conventional phase referencing of BL Lac at 5, 8, 15, and 
$22 \mathrm{GHz}$ were performed, with $\mathrm{J} 2153+4322$ and $\mathrm{J} 2218+4146$ as the reference sources and a cycle time of $80 \mathrm{~s}$. Following each phase referencing block, we had fast frequency-switching observations, just on BLLac, between $22-43 \mathrm{GHz}$ and $22-86 \mathrm{GHz}$, with $30 \mathrm{~s}$ per scan over a 15 minute block. These were bracketed by ionospheric calibration blocks, which consist of observations switching between the L-band receiver range (16IFs between 1.4 and $1.7 \mathrm{GHz}$ ), the wide band C-band receiver (16IFs between 3.9 and $7.9 \mathrm{GHz}$ ), and the K-band receiver (16IFs between 21.8 and $22 \mathrm{GHz}$ ), with $40 \mathrm{~s}$ of observing time at each band. For the switching observations the on-source time was $1.3 \mathrm{hr}$ for each frequency in the pair. The data reduction was in AIPS, following the standard path of correction for SEFD amplitudes, correction for Earth orientation parameters and correction for the ionosphere, based on GPS models.

\section{MULTI-FREQUENCY PHASE REFERENCING}

The SFPR method, which has been described in detail elsewhere (Dodson \& Rioja 2009; Rioja \& Dodson 2011; Rioja et al. 2011, 2014, 2015; Dodson et al. 2014), consists of two calibration steps. In a first step, the observations at the higherfrequency bands are calibrated using near-simultaneous (Dodson \& Rioja 2009; Rioja \& Dodson 2011; Rioja et al. 2014) or simultaneous (Dodson et al. 2014; Rioja et al. 2014, 2015) observations at a lower-frequency band, for each source. This is done for all frequency pairs which have an integer ${ }^{6}$ frequency ratio, by which the low-frequency calibration phase solutions are scaled. This dual frequency calibration step eliminates the common non-dispersive residual errors (e.g., tropospheric propagation effects and geometric errors) in the complex visibility output of the correlator, providing an increased signal coherence at the higher frequency. The second step of the calibration removes the remaining dispersive residual errors (i.e., instrumental and ionospheric propagation effects) using the interleaved observations of another source. This two-step calibration retains the astrometric signature of any source position shifts between the two frequencies in the interferometric phase observable. The Fourier transformation of the SFPR data set is the SFPR map, which conveys a bona-fide astrometric measurement of the relative separation or shift between the reference points in the images at the two frequencies, for the two sources. Results from SFPR analysis are to be found in the cited papers.

SFPR relies on the observation of a second (or multiple, as in the analysis of Rioja et al. 2015) calibrator. This can be some distance from the target, with successful demonstrations with separations as large as $11^{\circ}$ (Dodson \& Rioja 2009; Rioja et al. 2015), but there are sources for which one still struggles to find a suitable calibrator at the highest frequencies. This is the case for BL Lac where no millimeter-wavelength calibrator, for direct or reverse SFPR, could be found within $10^{\circ}$. Therefore we have attempted to achieve phase referencing in a similar fashion to SFPR, but without the second source. Our approach in this experiment is to calibrate all frequencies against a wellknown source with precise astrometric position, then solve for the residual delays for the target, across a wide frequency span. This allows us to measure the residual total electron content (TEC) in the target direction, which is used to produce an

\footnotetext{
6 For non-integer ratios see the analysis and discussions in Dodson et al. (2014).
}

ionosphere-free data set for all frequencies. This method we dub multi-frequency phase referencing (MFPR), as now our calibration scheme allows relative astrometry between the (mm) frequency bands corrected by observations at multiple $(\mathrm{cm})$ frequencies of the target.

\section{METHODS}

The prime calibration was against 3C 345, except for $86 \mathrm{GHz}$, where we had many missed scans and the data quality was very poor. For this frequency $3 \mathrm{C} 84$ was the prime calibrator and prime calibration could only be performed for the BR, KP, LA, OV, and PT antennas. Prime calibration removes the instrumental terms, plus all the atmospheric contributions in the direction of the calibrator, at the time of the observations. We handled the structural contribution from the source by hybrid mapping the data before astrometric calibration, to produce a reference image which is used in the analysis. The conventional phase referencing at $\mathrm{cm}$-wavebands was calibrated following standard procedures and the detailed interpretation will be reported elsewhere S. Molina (2017, in preparation).

For the ionospheric correction blocks we use the delay (only) from each IF to measure the TEC contribution on the line of sight toward BLLac, as a function of time. This is a measurement of the residual ionospheric contribution, after correction with the GPS data and the subtraction of the TEC in the direction of the prime calibrator, at the time of that scan. We fitted a linear slope in $\nu^{-2}$ to the semi-simultaneous delay measurements (i.e., such that $\tau(\nu)=\tau_{\text {trop }}+\tau_{\text {iono }} \nu_{\mathrm{GHz}}^{-2}$ ) and calculate the residual TEC contribution as a function of time, for that line of sight, using $\triangle \mathrm{TEC}=0.75 \tau_{\text {iono }}$. Here $\tau(\nu)$ are the measured delays as a function of frequency for one block of ionospheric calibration observations, $\tau_{\text {trop }}$ is the non-dispersive delay (from both clock and tropospheric contributions), $\tau_{\text {iono }}$ is the ionospheric delay (at $1 \mathrm{GHz}$ ), $\nu_{\mathrm{GHz}}$ is the frequency in $\mathrm{GHz}$, and $\triangle \mathrm{TEC}$ is the deduced residual ionospheric contribution in TEC units (TECU), to the line of sight of the target. We are assuming, as we have corrected the instrumental terms $\left(\tau_{\text {inst }}\right)$ using the prime calibration, that these are constant during the experiment and therefore can be ignored in this analysis. The derived $\triangle \mathrm{TEC}$ is then used to calculate the ionospheric contribution for each IF, at each time interval. The frequencydependent delay can directly be calculated, but note that the sign of the phase has to be reversed, as the ionospheric contribution is a group delay, not a phase delay. Finally we solved for the (ionosphere-free) delay, rate, and phase on the (ionosphere-corrected) $22 \mathrm{GHz}$ data and applied these solutions, suitably scaled by the frequency ratio, to the ionospherecorrected 43 and $86 \mathrm{GHz}$ data. As this calibration scheme uses low-frequency calibration blocks, either side of the highfrequency science observations, and these are to correct for the ionospheric contributions to the atmosphere, we dubbed these IonospheriC Excision blocks (ICE-blocks). This recognizes the commonality with the so-called geodetic block calibration schemes that dealt with the static tropospheric contributions (Brunthaler et al. 2005; Honma et al. 2008; Reid \& Honma 2014).

Our expectations were that, having corrected for the ionospheric and the tropospheric contributions, we should be left with the high-frequency data sets astrometrically aligned to the $22 \mathrm{GHz}$ data set. However, as will be discussed in the results, we found that our initial calibration scheme was 


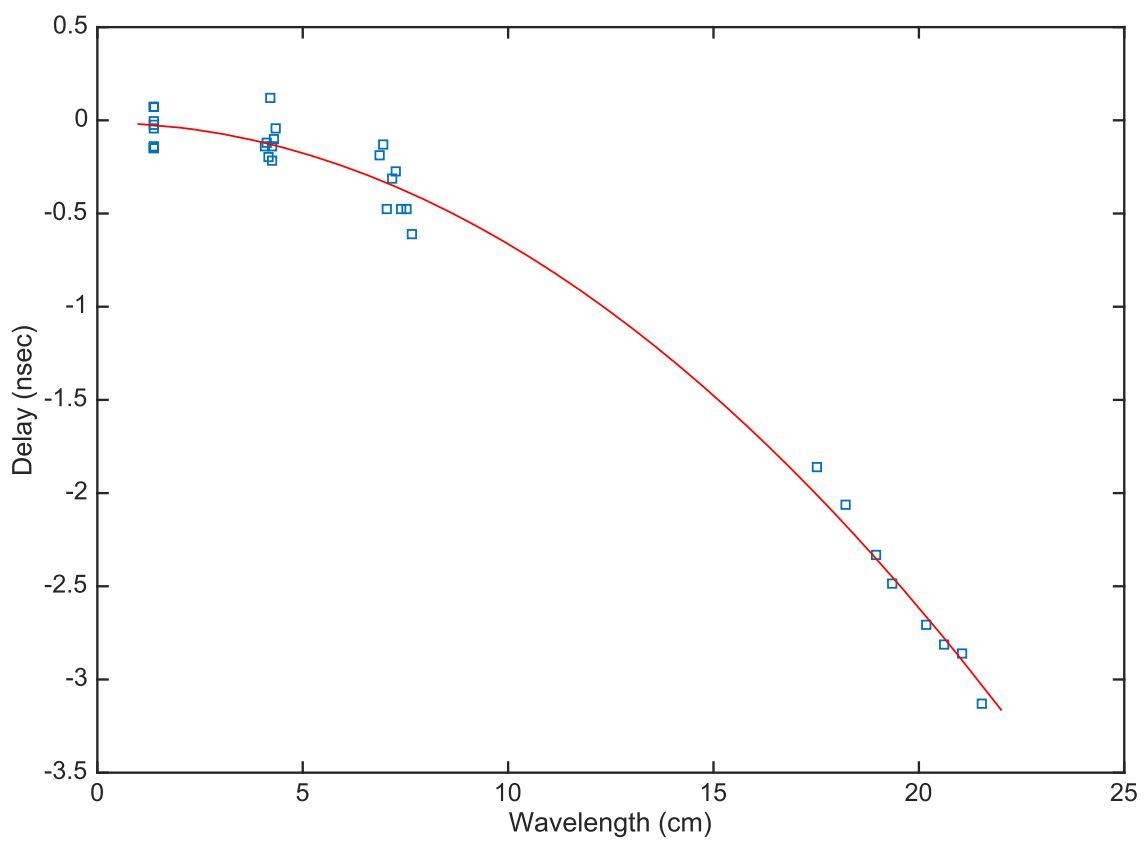

Figure 2. A typical delay $\tau(\nu)$ for one particular antenna (BR) and 2 min ICE-block scan (UT 05:56), as a function of wavelength. It shows the curvature (following $\nu^{-2}$ ) that directly measures the $\triangle \mathrm{TEC}$ residual (in this case $-4.4 \mathrm{TECU}$, indicated with the solid line), for that solution interval and for that antenna, in the line of sight of the target.

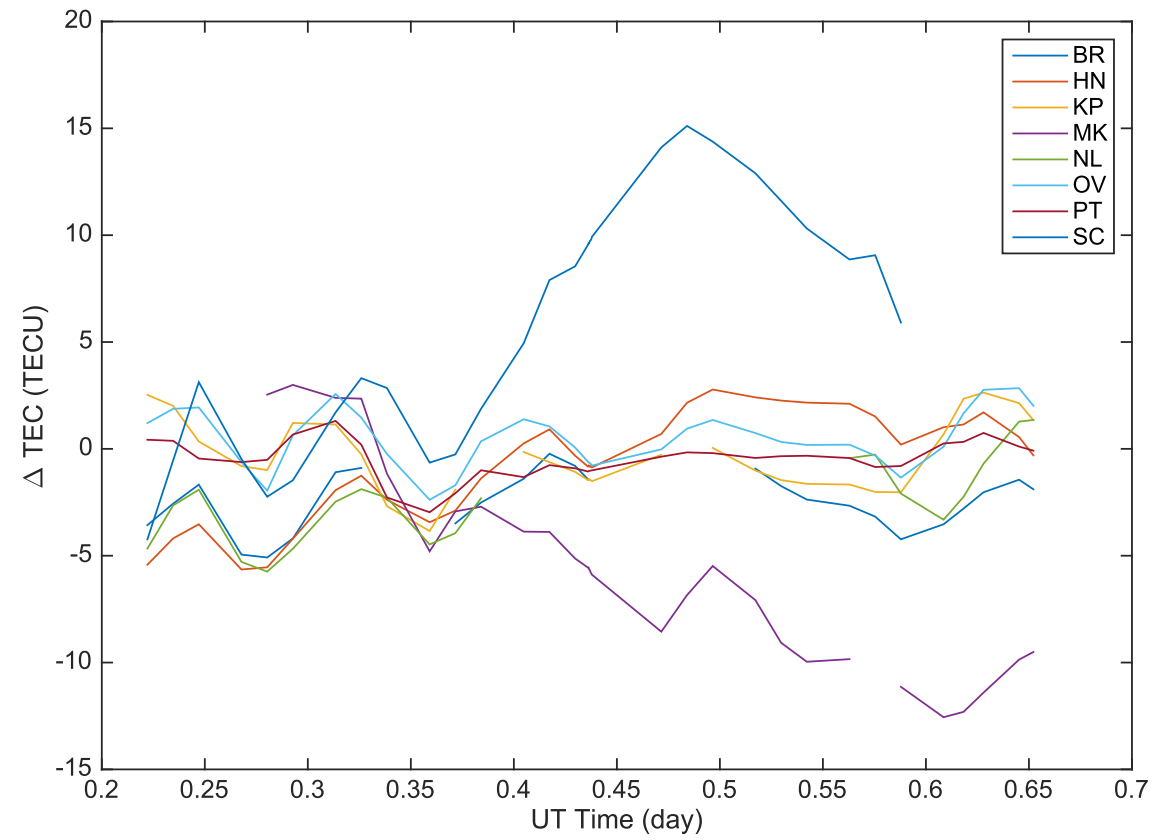

Figure 3. The $\triangle \mathrm{TEC}$ residuals for all antennas across the duration of the experiment. The values for most antennas range between $\pm 5 \mathrm{TECU}$, as would be expected for data that have been corrected with the default TEC maps, using TECOR. The antennas MK and SC show the largest deviations. Errors in individual measurements are typically 0.1 TECU.

inadequate. In our observations of $3 \mathrm{C} 345$ we have a gap of about five minutes between the prime calibration observations at 22 and $43 \mathrm{GHz}$, which was used for a pointing correction. An even longer gap existed between the 22 and $86 \mathrm{GHz}$ prime calibrator scans. Therefore we could not align the phase of these scans to a common point in time. This caused an unknown phase offset to be introduced between the data at $22 \mathrm{GHz}$ and higher frequencies, in the observation of the target source at the target frequencies. Therefore prime calibration should have included a fast frequency-switching scan on the calibrator as well as the target; this will be included in future observations. How we resolved this problem for these observations is discussed in Section 5.2.

\section{RESULTS}

\subsection{Ionospheric Measurements}

Each ICE-block consists of multiband observations at 1.4-1.7, $3.9-7.9$, and $22 \mathrm{GHz}$, that is $21-18 \mathrm{~cm}, 8-4 \mathrm{~cm}$, and $1.3 \mathrm{~cm}$. These bracket the fast frequency-switching observations of the target source, which either consist of $22-43$ or $22-86 \mathrm{GHz}$ blocks. The ICE-blocks are calibrated following standard 

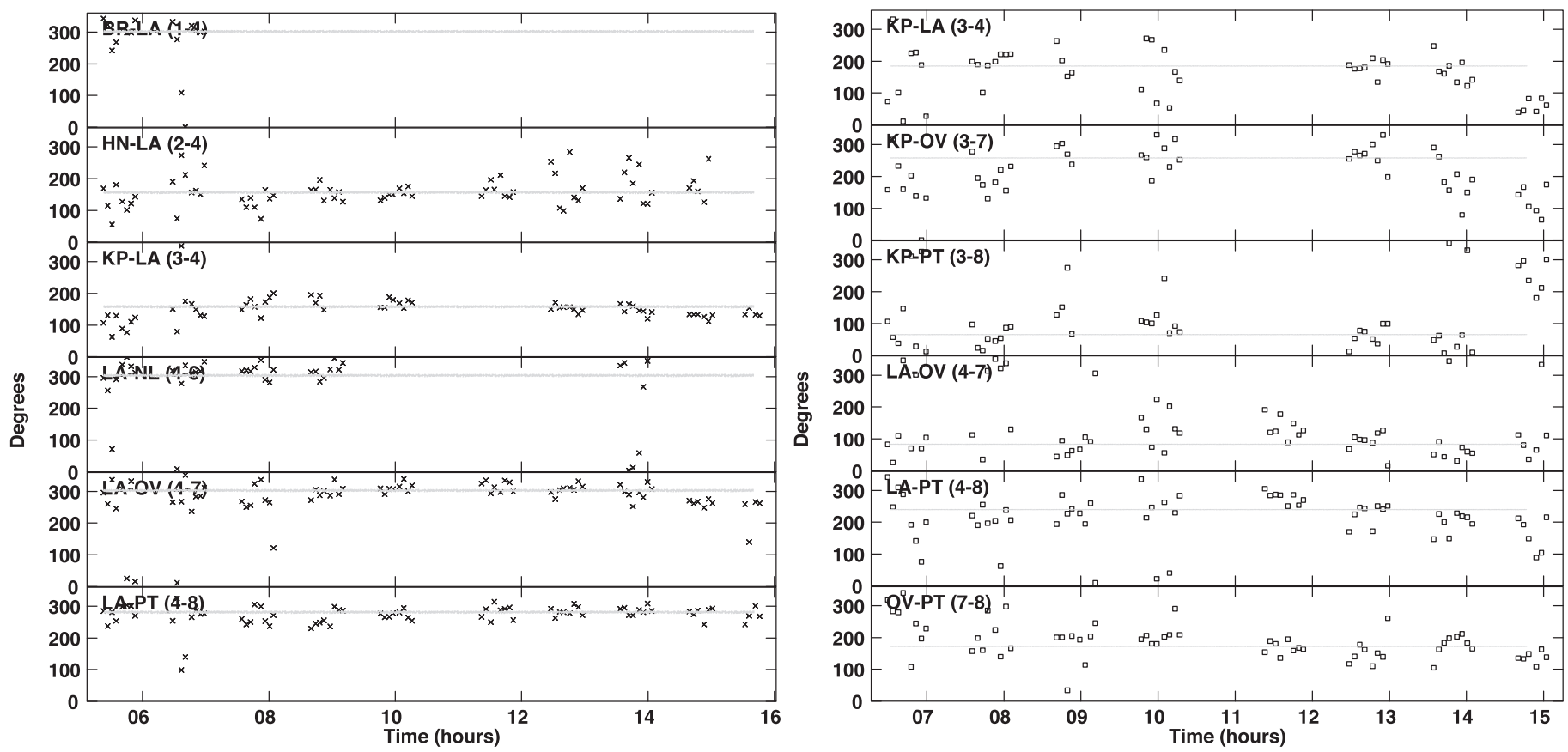

Figure 4. Ionosphere- and troposphere-corrected visibilities on BL Lac showing the dominant constant phase offset (indicated with light gray lines) arising from the non-simultaneous observation of the prime calibrator, for (left) $43 \mathrm{GHz}$ with baselines to Los Alamos (LA) only and (right) $86 \mathrm{GHz}$ with all baselines that could be calibrated.

procedures and the measured delays for each IF fitted to derive $\triangle T E C$. Figure 2 shows the delays as a function of frequency for one scan on one antenna. Figure 3 shows the derived $\triangle T E C$ for all antennas during this experiment. The fitting errors can be used to estimate the measurement precision of the $\triangle \mathrm{TEC}$ residuals. The error level was $\sim 0.1$ TECU, if one excluded MK and SC (which were not included in the final MFPR imaging, due to the poor quality of the data) and 0.2 TECU if all antennas were included. 0.1 TECU would contribute 2.2 of phase at $22 \mathrm{GHz}$, which would be scaled up to $3^{\circ} .3$ and $8^{\circ} .2$ of phase noise at 43 and $86 \mathrm{GHz}$, respectively (Rioja \& Dodson 2011).

\subsection{Constant Phase Offset Measurement and Astrometric Results}

After subtraction of the calculated ionospheric delays and phases from the 22,43 , and $86 \mathrm{GHz}$ data sets, we selfcalibrated the $22 \mathrm{GHz}$ BL Lac data against a hybrid map of the source, scaled the solutions by the frequency ratio, and used these to correct the higher frequencies. Thus the ionospheric and tropospheric contributions were removed. However, because the initial prime-calibrations at different frequencies were not at close points in time, there is an introduced constant, but unknown, phase at each station. This is shown in Figure 4 for 43 and $86 \mathrm{GHz}$. The Fourier inversion of these data, after excluding antennas MK and SC plus the low elevation data (the first and last hour), gives us our initial image. However this image did not recover the source structure, because of the error in the initial calibration chain.

To remove a constant phase yet not lose the astrometric signal we trialed all possible models (point sources on a $10 \mu$ as grid within 0.5 mas of the phase center), generating a single phase correction for each antenna for the whole data set, using self-calibration. Using this calibration table we repeated the imaging and inspected the peak flux, the residual rms, and the dynamic range as a function of the model. The peak in the dynamic range, plotted in Figure 5, gives an astrometric alignment of $-18 \pm 10,+12 \pm 10 \mu$ as, based on the $95 \%$ confidence limits of a 2D second-order polynomimal fit to the surface. Note that the dynamic ranges achieved (a maximum of 20) for a single-point source fit are much less than those for the self-calibrated hybrid images, for which we had a dynamic range of $\sim 200$. The most precise test, however, is the alignment of the location of the peak flux and that of the trial model. The minimum absolute offset in the alignment gives an astrometric result of $-8 \pm 5,20 \pm 6 \mu \mathrm{as}$, based on the $95 \%$ confidence limits of two orthogonal 1D second-order polynomial fits to the surface, as shown in Figure 6. The precision of the alignment is approximately double that of the precision from the peak in the dynamic range so we adopt this value as our astrometric result. The image of BL Lac made with the phase corrections for an offset of $-8 \pm 5,20 \pm 6 \mu$ as is shown in Figure 7. Unfortunately the $86 \mathrm{GHz}$ data were of too poor quality to produce useful results. In a subsequent paper we will extend our analysis to $86 \mathrm{GHz}$ using a better data set from our sample of blazars included in this project.

To test the physicality of our results we compared the derived $22-43 \mathrm{GHz}$ core-shift measurement to the preliminary results from the centimeter-wavelength phase referencing. We performed conventional phase referencing for the 4.8, 8.4, 15, and $22 \mathrm{GHz}$ (see Figure 8) data against the compact calibrator $\mathrm{J} 2153+4322$ (Figure 9), which is $2^{\circ}$ from BL Lac. The images are similar and in agreement with published observations. We restored the BLLac images to the common beamsize of the $22 \mathrm{GHz}$ image used as the reference for the FPT, to avoid blending issues that otherwise shift the apparent positions. We measured the relative positions of the peak of emission (compared to that of the referenced source) at all frequencies and found a systematic shift across the sky between 4.8 and $22 \mathrm{GHz}$, with $r_{0}$ of 5.3 mas $\mathrm{GHz}^{\kappa}, \kappa$ of -0.99 and an offset of $45 \mu$ as. These parameters would lead to a prediction of $120 \pm 30 \mu$ as for the core-shift between 22 and $43 \mathrm{GHz}$. This 


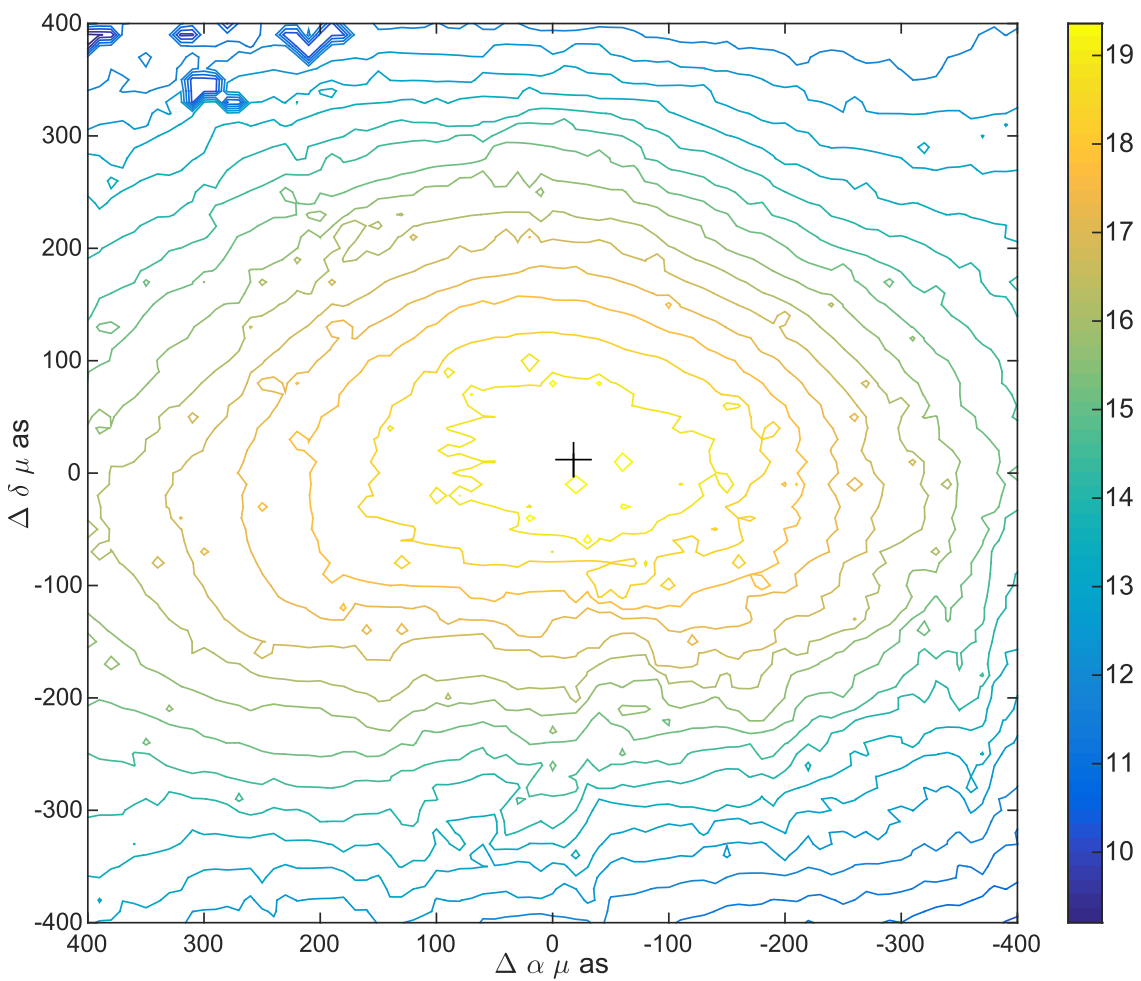

Figure 5. The dynamic range as a function of the $\Delta \alpha, \Delta \delta$ of the input model. Also marked is the peak value, at $-18,12 \mu$ as, and the error bounds of $\pm 10 \mu$ as.

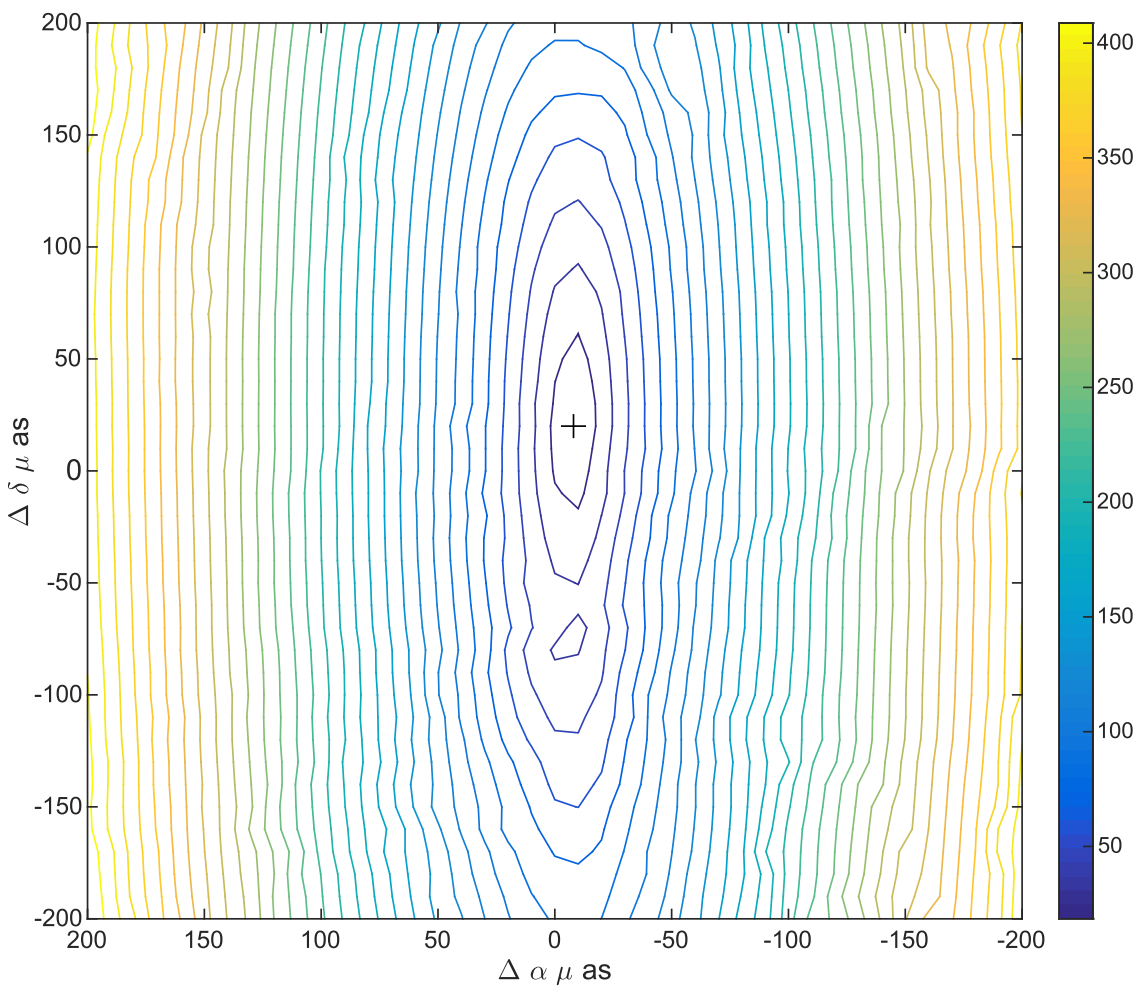

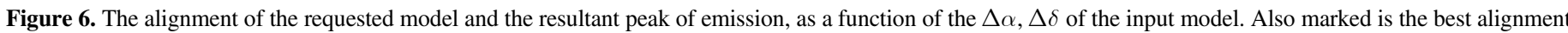
at $-8,20 \mu$ as and the error bounds of $\pm 5,6 \mu$ as.

is much greater than found. Additionally we fitted for all frequencies, including $43 \mathrm{GHz}$. The best fit was obtained with $r_{0}$ of $7.7 \mathrm{mas} \mathrm{GHz}^{\kappa}, \kappa$ of -1.32 and an offset of $190 \mu$ as. This fit, although valid, would imply a significant departure from the expected value of $\kappa \simeq-1$, for the case of equipartition between jet particle and magnetic field energy densities (e.g.,
Lobanov 1998), as found at cm-wavebands in BL Lac and other multiple sources (i.e., O'Sullivan \& Gabuzda 2009; Hada et al. 2011; Sokolovsky et al. 2011). It would also imply a large error in the astrometric position of BLLac and/or J2153 +4322 , which is unlikely. Figure 10 shows the location of the peaks of emission, along with predictions from the two models. 


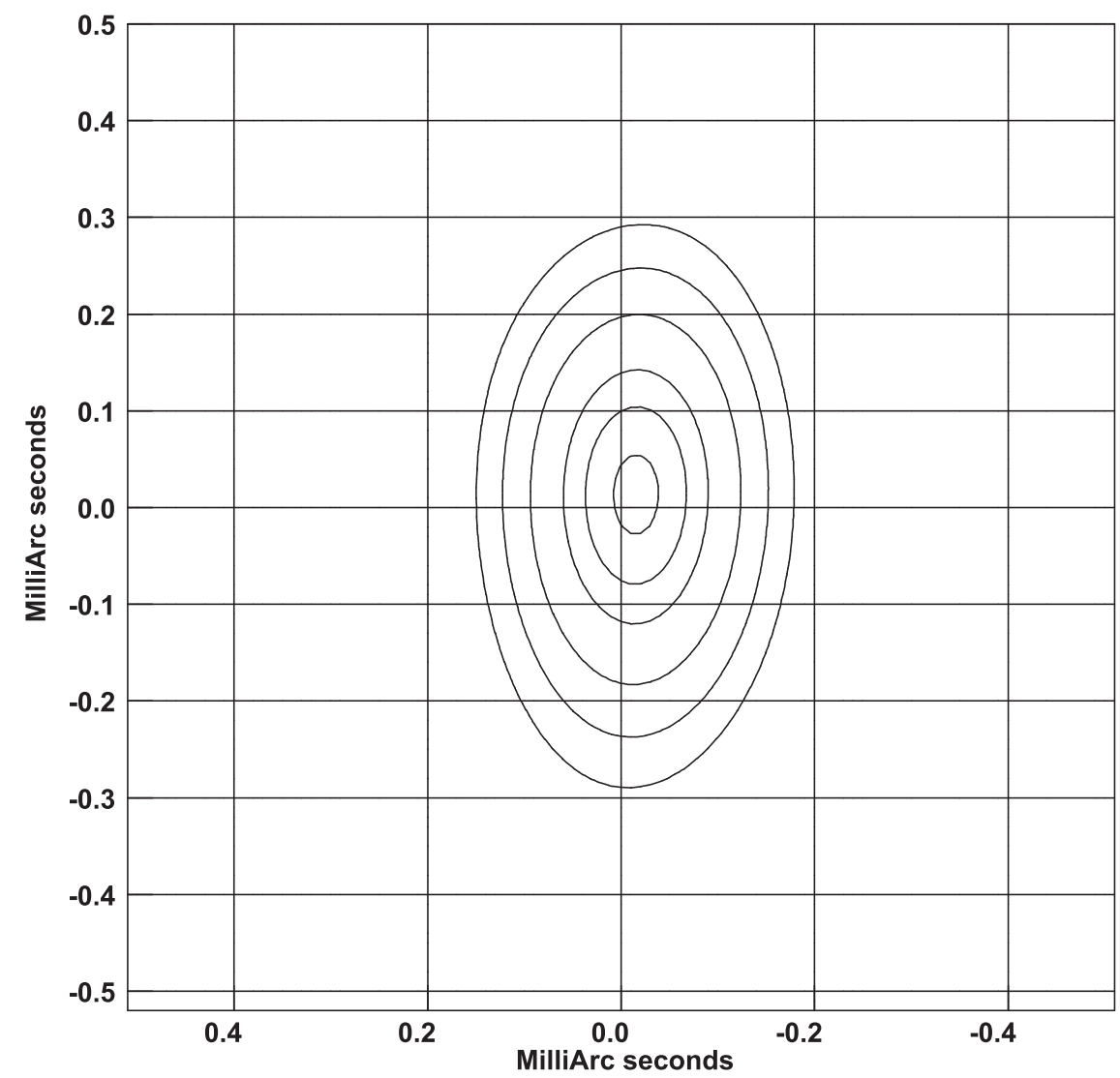

Figure 7. Image of BL Lac at $43 \mathrm{GHz}$, multi-frequency phase referenced to the $22 \mathrm{GHz}$ data. The offset from center $(-8,+20 \mu$ as) is the bona-fide astrometric core shift between 22 and $43 \mathrm{GHz}$. Contours are $60 \%, 70 \%, 80 \%, 95 \%$, and $99 \%$ of the peak flux (3.3 Jy/beam). The restoring beam size is $0.25 \times 0.34$, PA $-22^{\circ}$.

\section{DISCUSSIONS}

We have demonstrated that the effects of the atmosphere can be calibrated in a step-wise manner, by decomposing them into dispersive and non-dispersive contributions. We have included for the first time a wide-band measurement of the residual ionospheric contributions to VLBI data, for the line of sight of the target. Our measurement of the ionosphere, derived from the group delay curvature, is a development of the standard geodetic two-point fit (at 2.4 and $8.4 \mathrm{GHz}$ ) approach (Williams et al. 1979; Sovers et al. 1998), whereas we fit the data over multiple frequencies. We performed simple simulations with typical values for $\triangle$ TEC and $\Delta \ell$, the residual TEC and path length, and measurement errors of $0.1 \mathrm{~ns}\left(1^{\circ}\right.$ phase error across $16 \mathrm{MHz}$ ). We explored the required frequency span to be able to predict accurate values for $\triangle \mathrm{TEC}$, and found that the lower frequencies are the most crucial. At least $2.4 \mathrm{GHz}$ would be required, and $1.4 \mathrm{GHz}$ would be preferred. Using our data we investigated the achievable reliability using just the new VLBA wideband C-band and K-band data, and found the accuracy in the determined $\triangle$ TEC to be $\sim 1$ TECU. Such levels are those expected (i.e., by scaling with $\left.\left(\nu_{1} / \nu_{2}\right)^{2}\right)$ and would be sufficient mitigate the ionospheric contribution to the errors in the astrometry of methanol masers at $6.7 \mathrm{GHz}$.

The FPT has been thoroughly demonstrated previously (Rioja et al. 2015, and references therein), therefore we would expect the approaches taken here to be successful. The conditions for which we may expect this to break down are: where instrumental terms are not stable, introducing a time variable non-dispersive term that is not a function of $\nu^{-2}$; and where there are baseline-dependent terms introduced, for example, by having a poor model of the prime calibrator. For our analysis we do not believe the first of these are issues, as the VLBA has extremely stable instrumental terms.

We addressed the issue of the baseline-dependent phase terms introduced by the prime-calibrator by using a hybrid model in the initial fringe fitting stage. This worked well for the core-dominated source 3C 345, but failed for the more complex structure of $3 \mathrm{C} 84$, where there were insufficient data at $86 \mathrm{GHz}$ to constrain the model. We note that any core-shift in the prime calibrator will appear as a constant phase introduced between the two frequencies, as it will arise from a single scan. Therefore it will be absorbed into the constant phase corrections discussed next.

To remove the phase introduced between the two frequencies from the prime-calibration we performed a grid search to find the best constant phase that matched the data. Our approach of testing for model stability is similar to super-resolution. In super-resolution one adjusts the model to the uv-data to locate the best-fitting location using a minimization method, thereby producing a positional accuracy greater than the resolution. In our case we are testing the fit of a model to the uv-data, with a cycle of self-calibration, in a stepwise fashion, over the parameter space. This aspect of our analysis is perhaps the most innovative, but also suffers from the limitations in our minimization methods. We have no mechanism to measure the co-variance between the fitted parameters of source position and constant station-based phase offset. If the experiment had been a "snapshot" these two would be degenerate; however, as the observations spanned $8 \mathrm{hr}$ this degeneracy is broken. We estimated our errors from the fitting to the $2 \mathrm{D}$ surface of the 


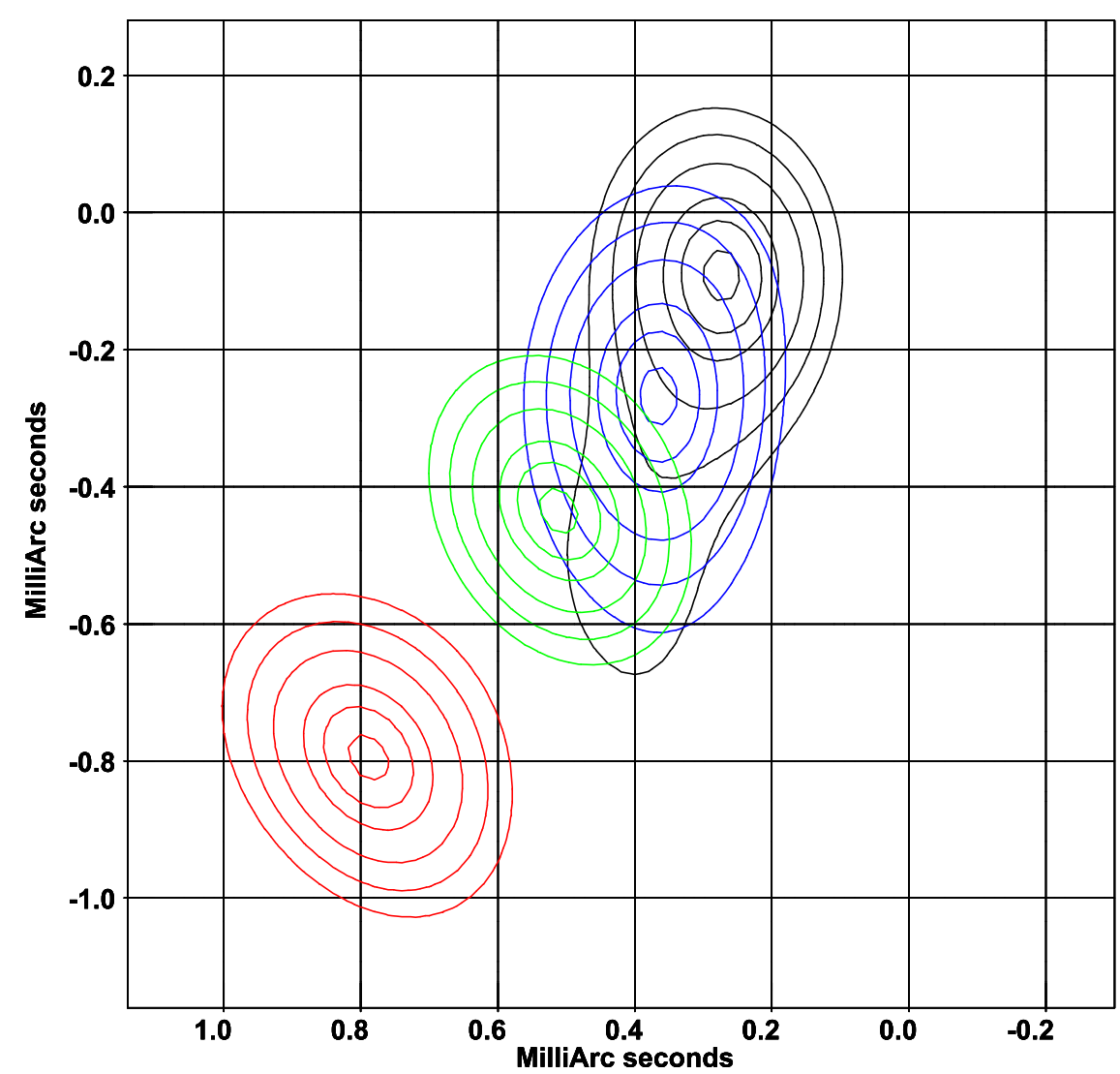

Figure 8. Overlaid astrometrical-registered images of BL Lac for the nominal phase center of 22:02:43.291 and 42:16:39.980 in R.A. and decl., derived using conventional methods and phase referenced to J2153+4322. Four frequencies are plotted: 4.8, 8.4, 15.2, and $21.9 \mathrm{GHz}$ in red, green, blue, and black respectively. Contours are $60 \%, 70 \%, 80 \%, 95 \%$, and $99 \%$ of the peak flux for each frequency, which are 1.6, 3.7, 2.1, and 1.6 Jy/beam.

results. The use of independent measures for these surfaces reduces the possibility of degeneracy. That the maximum dynamic range (and peak flux and minimum residual rms) align with those of the minimum absolute offset in the alignment gives confidence in our results.

Our conclusions would be significantly strengthened if we were able to produce a joint analysis of the $22-43$ and $86 \mathrm{GHz}$ data. However, this was not possible, because of the poor quality of the observations at $86 \mathrm{GHz}$, due to technical issues. These data could not even be self-calibrated to produce acceptable images. Therefore we believe the residuals probably arise from baseline-based (rather than station-based) contamination from the prime-calibrator scan, which cannot be corrected for by our procedures.

Ideally we would compare our new method with results from conventional phase referencing for the same source. However if conventional phase referencing was possible at these frequencies we would not have needed to develop these new methods. We used the measurement of the core-shifts made at lower frequencies with conventional phase referencing, and extrapolated these results to compare with our MFPR measurement made at millimeter-wavelengths. However these can provide only the Blandford \& Königl core-shift; our expectation is that the higher frequencies would have smaller than predicted coreshifts. This is consistent with what we discover, as shown in Figure 10. Consequently we cannot use this approach to validate our method.

We attempted to derive the core shift following the approach of aligning optically thin features, but we could not get sufficiently accurate results from our own data. Optically thin features tend to be of lower surface brightness and therefore it is difficult to accurately determine the centroids (Hovatta et al. 2014). Nevertheless cross-correlation of VLBA images first by O'Sullivan \& Gabuzda (2009), and second by Gómez et al. (2016) (with observations made a few months after ours in 2013 November) have determined a core-shift of $30 \pm 20$ $\mu$ as and $21 \mu$ as, respectively, between 22 and $43 \mathrm{GHz}$. This is in close agreement with the value we find from the MFPR analysis, which provides extra support for the reliability of our new method. The alignment reported by O'Sullivan \& Gabuzda (2009) is based on the cross-correlation of images at frequencies between 5 and $43 \mathrm{GHz}$. They find an average core-shift of the $22 \mathrm{GHz}$ position, referenced to $43 \mathrm{GHz}$, of $40 \pm 20 \mu$ as, whereas we have compared with only the measurement between 22 and $43 \mathrm{GHz}$. All measurements are consistent within the errors. They fitted their cross-correlation alignments to obtain a value of $\kappa$ (following our definition) of -1.01 between 5 and $43 \mathrm{GHz}$, which would at first sight appear to be in contradiction with our results. However the functional expression $\left(\left(\nu_{2}-\nu_{1}\right) /\left(\nu_{1} \nu_{2}\right)\right)$ that they fit is not very sensitive to deviations between 22 and $43 \mathrm{GHz}$. Replacing all of their $43 \mathrm{GHz}$ measurements with the $22 \mathrm{GHz}$ values (i.e., inserting a zero core-shift between 22 and $43 \mathrm{GHz}$ ) and repeating the fitting reproduces the published result, within errors. Furthermore, in our analysis, we found that blending of components within the uniform weighted beam at the low frequencies distorted our results, which is why we restored with the superresolved beam of the $22 \mathrm{GHz}$. This important issue was not addressed in the O'Sullivan \& Gabuzda (2009) analysis. 


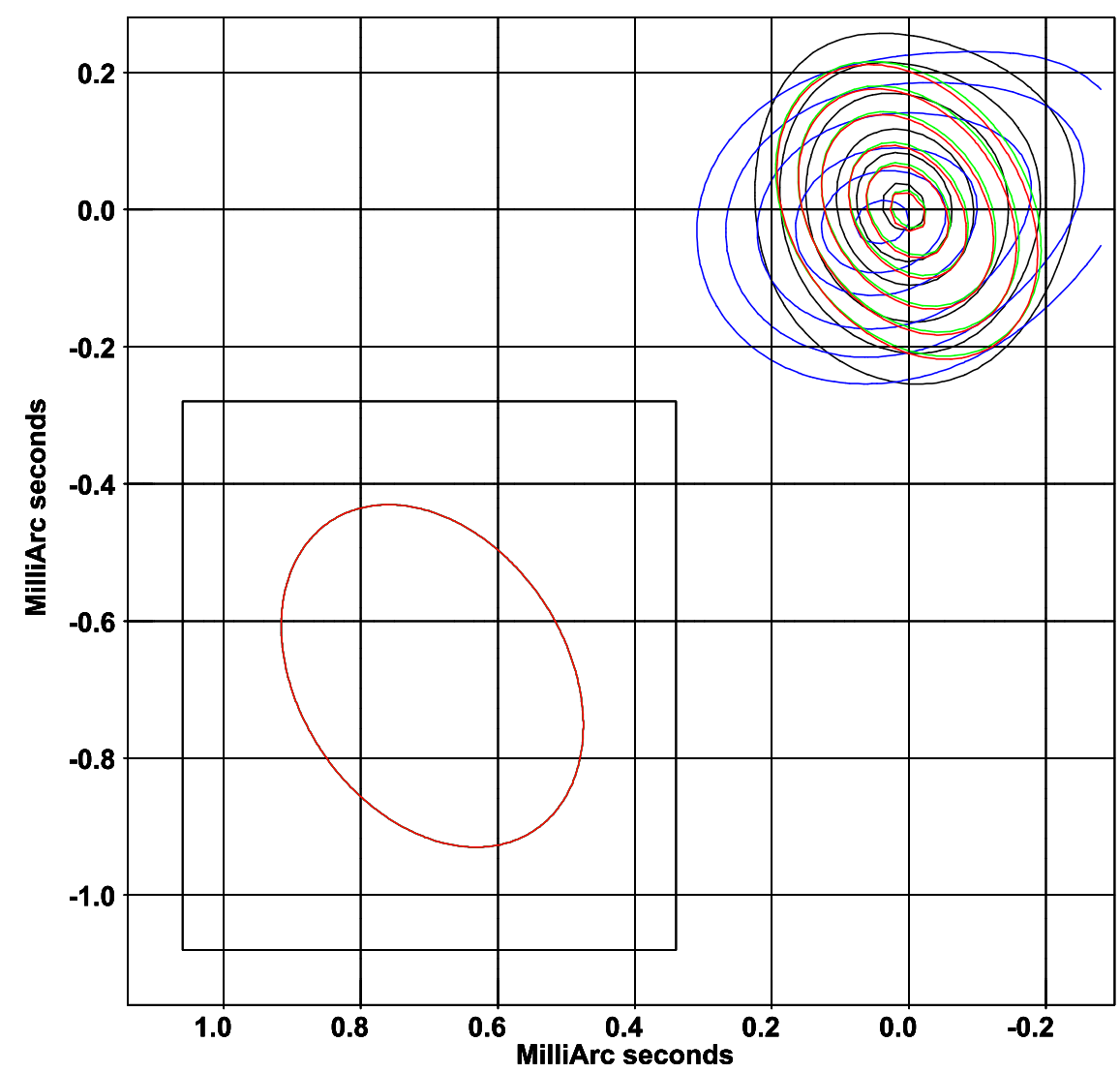

Figure 9. Overlaid self-calibrated images of J2153+4322 for the nominal phase center of 21:53:50.959 and 43:22:54.500 in R.A. and decl. All images are restored with the $22 \mathrm{GHz}$ beam parameters of the image $\left(0.57 \times 0.72, \mathrm{PA}-26^{\circ}\right)$ used to reference the $43 \mathrm{GHz}$ data. Four frequencies are plotted: $4.8,8.4,15.2$, and $21.9 \mathrm{GHz}$ in red, green, blue, and black respectively. Contours are $60 \%, 70 \%, 80 \%, 95 \%$, and $99 \%$ of the peak flux for each frequency, which are $0.25,0.28,0.12$, and 0.08 Jy/beam.

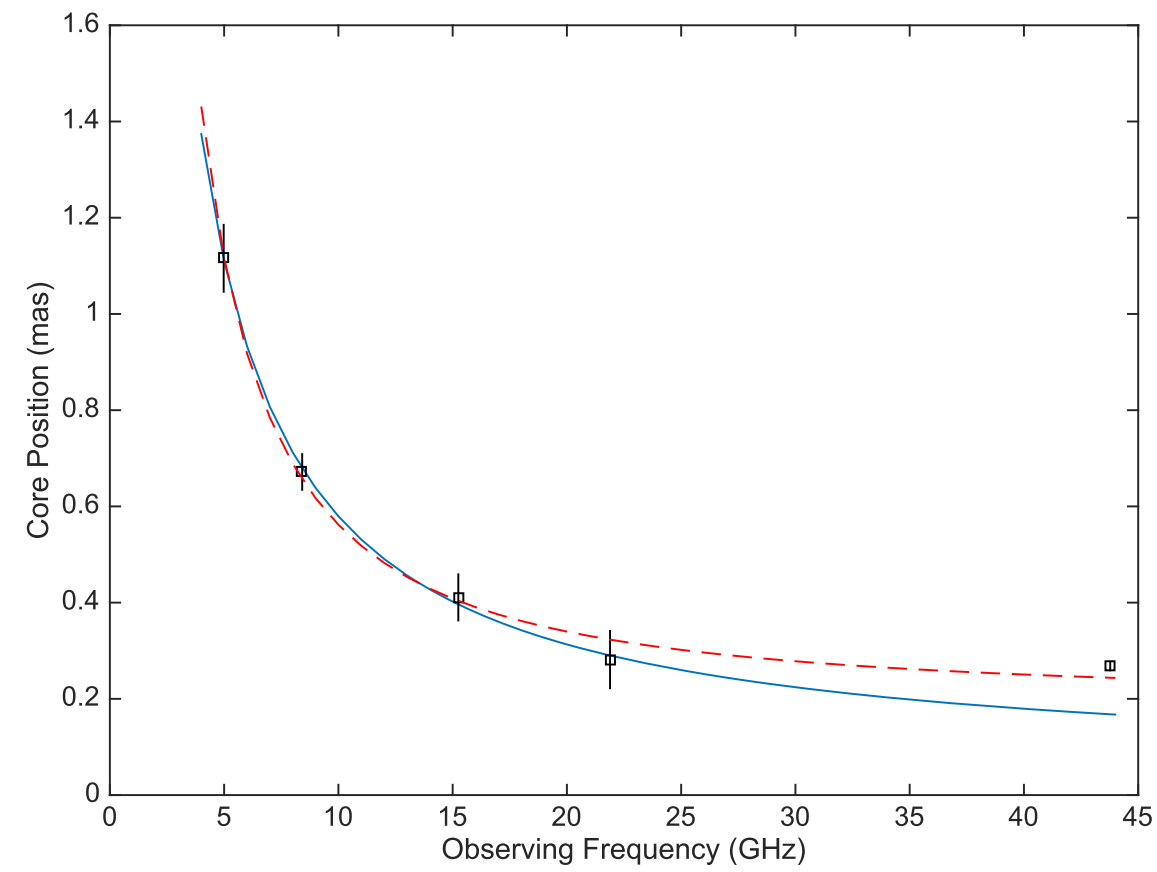

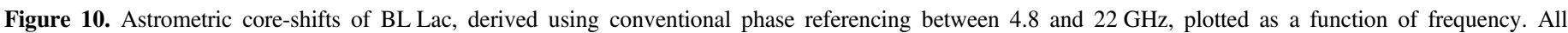

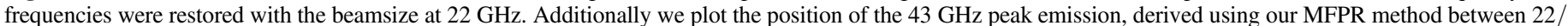

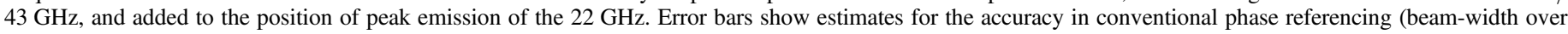

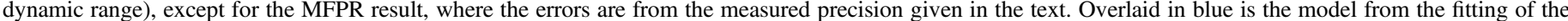

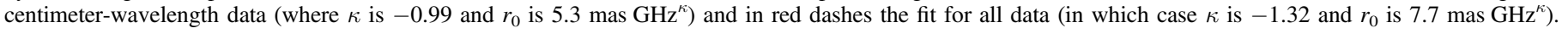


This multi-frequency approach is one of two methods we are currently developing to improve astrometric calibration of the ionospheric contributions, the other being to use multiple calibrators around the target and to solve for the spatial structure of the atmosphere, which we call MultiView (Rioja et al. 2009, 2016). This method, which requires more calibrators rather than fewer, simultaneously solves for a 2D ionospheric and tropospheric phase screen over the array, and will be extremely suitable for VLBI stations with multiple beams, such as SKA and ASKAP.

\section{CONCLUSIONS}

We have presented a development of the SFPR method, which we call MFPR. This has been used to measure the coreshift (or the $\lambda$-astrometry) for BL Lac, between 22 and $43 \mathrm{GHz}$. The MFPR method presented here involved a measurement of the $\triangle$ TEC on the line of sight of the target, with a precision of 0.1 TECU. Once the data are corrected for the ionosphere, the tropospheric correction is measured at the lower frequency and applied to the higher frequency. The high-frequency data should then be registered to the low-frequency data using FPT. Our data required an additional step, to minimize over a constant phase offset introduced by the prime calibration, which can be avoided in future observations with improved scheduling. After these steps the astrometric offset between the two frequencies is the bona-fide core-shift, which has been derived from observations of a single source by careful calibration of the atmospheric contributions.

This new method opens up a large number of possibilities for astrometric analysis in mm-VLBI. Conventional phase referencing is not possible for mm-VLBI, and for a significant number of sources, such as BL Lac, a suitable calibrator as required for SFPR cannot be found. The method of alignment of optically thin components suffers from both questionable validity, and a requirement of high sensitivity to detect low surface brightness features. MFPR bypasses all of these issues, requiring only a detectable target source at the lower frequencies. With simultaneous observations this method will be applicable to $\mathrm{mm}$ and sub-millimeter-wavelengths.

The results from the MFPR measure a core-shift for BL Lac of $-8 \pm 5,20 \pm 6 \mu$ as between 22 and $43 \mathrm{GHz}$. This is significantly less than the prediction from measurements of the core-shift at centimeter-wavelengths, but in line with both the theoretical expectations and other work.

Further analysis, to be published in S. Molina (2017, in preparation), will improve the initial measurement of the coreshift at centimeter-wavelengths, allowing us to deduce if we are truly uncovering deviations from the Blandford \& Königl model. If so this would be the first detection of the predicted association of the mm-VLBI core with a recollimation shock responsible for the $\gamma$-ray emission in blazar jets, in agreement with the findings of Gómez et al. (2016). The fact that the calibration of the $86 \mathrm{GHz}$ showed promise but was defeated by an unusually large antenna failure rate gives us confidence that we will be able to perform MFPR at $86 \mathrm{GHz}$ in future demonstrations.

We would like to especially acknowledge the numerous conversations with Richard Porcas about the best approaches for the analysis, and the referee for comments that improved the paper. The work at IAA-CSIC is supported by the Spanish Ministerio de Economía y Competitividad grant AYA201340825-P. The VLBA is operated by National Radio Astronomy
Observatory and is a facility of the National Science Foundation operated under cooperative agreement with Associated Universities Inc.

\section{REFERENCES}

Alef, W. 1988, in IAU Symp. 129, The Impact of VLBI on Astrophysics and Geophysics, ed. M. J. Reid \& J. M. Moran (Cambridge, MA: Kluwer), 523 Aloy, M.-Á., Martí, J.-M., Gómez, J.-L., et al. 2003, ApJL, 585, L109

Asada, K., \& Nakamura, M. 2012, ApJL, 745, L28

Asada, K., Nakamura, M., Doi, A., Nagai, H., \& Inoue, M. 2014, ApJL, 781, L2

Beasley, A. J., \& Conway, J. E. 1995, in ASP Conf. Ser. 82, Very Long Baseline Interferometry and the VLBA, ed. J. A. Zensus, P. J. Diamond, \& P. J. Napier (San Francisco, CA: ASP), 327

Blandford, R. D., \& Königl, A. 1979, ApJ, 232, 34

Brunthaler, A., Reid, M. J., Falcke, H., Greenhill, L. J., \& Henkel, C. 2005, Sci, 307,1440

Casadio, C., Gómez, J. L., Grandi, P., et al. 2015a, ApJ, 808, 162

Casadio, C., Gómez, J. L., Jorstad, S. G., et al. 2015b, ApJ, 813, 51

Chatterjee, R., Marscher, A. P., Jorstad, S. G., et al. 2011, ApJ, 734, 43

Daly, R. A., \& Marscher, A. P. 1988, ApJ, 334, 539

Dodson, R., \& Rioja, M. J. 2009, arXiv:0910.1159

Dodson, R., Rioja, M. J., Jung, T.-H., et al. 2014, AJ, 148, 97

Fromm, C. M., Perucho, M., Ros, E., Savolainen, T., \& Zensus, J. A. 2015, A\&A, 576, A43

Gómez, J. L., Lobanov, A. P., Bruni, G., et al. 2016, ApJ, 817, 96

Gómez, J. L., Martí, J. M., Marscher, A. P., Ibáñez, J. M., \& Alberdi, A. 1997, ApJL, 482, L33

Gómez, J. L., Marti, J. M. A., Marscher, A. P., Ibanez, J. M. A., \& Marcaide, J. M. 1995, ApJL, 449, L19

Guirado, J. C., Marcaide, J. M., Alberdi, A., et al. 1995, AJ, 110, 2586

Hada, K., Doi, A., Kino, M., et al. 2011, Natur, 477, 185

Honma, M., Tamura, Y., \& Reid, M. J. 2008, PASJ, 60, 951

Hovatta, T., Aller, M. F., Aller, H. D., et al. 2014, AJ, 147, 143

Jorstad, S., \& Marscher, A. 2016, Galax, 4, 47

Jorstad, S. G., Marscher, A. P., Smith, P. S., et al. 2013, ApJ, 773, 147

Königl, A. 1981, ApJ, 243, 700

Kovalev, Y. Y., Lobanov, A. P., Pushkarev, A. B., \& Zensus, J. A. 2008, A\&A, 483, 759

Kutkin, A. M., Sokolovsky, K. V., Lisakov, M. M., et al. 2014, MNRAS, 437, 3396

Lara, L., Alberdi, A., Marcaide, J. M., \& Muxlow, T. W. B. 1994, A\&A, 285, 393

Lobanov, A. P. 1998, A\&A, 330, 79

Marscher, A. P. 2009, in ASP Conf. Ser. 402, Approaching Micro-Arcsecond Resolution with VSOP-2: Astrophysics and Technologies, ed. Y. Hagiwar et al. (San Francisco, CA: ASP), 194

Marscher, A. P. 2012, IJMPS, 08, 151

Marscher, A. P., Jorstad, S. G., D' Arcangelo, F. D., et al. 2008, Natur, 452, 966 Marscher, A. P., Jorstad, S. G., Gómez, J.-L., et al. 2002, Natur, 417, 625

Marscher, A. P., Jorstad, S. G., Larionov, V. M., et al. 2010, ApJL, 710, L126

Martí, J. M., Perucho, M., \& Gómez, J. L. 2016, ApJ, 831, 163

Martí-Vidal, I., Marcaide, J. M., Alberdi, A., et al. 2011, A\&A, 533, A111

Mizuno, Y., Gómez, J. L., Nishikawa, K.-I., et al. 2015, ApJ, 809, 38

Molina, S. 2016, AJ

O'Sullivan, S. P., \& Gabuzda, D. C. 2009, MNRAS, 400, 26

Perucho, M., Bosch-Ramon, V., \& Khangulyan, D. 2010, A\&A, 512, L4

Reid, M. J., \& Honma, M. 2014, ARA\&A, 52, 339

Rioja, M., \& Dodson, R. 2011, AJ, 141, 114

Rioja, M., Dodson, R., Malarecki, J., \& Asaki, Y. 2011, AJ, 142, 157

Rioja, M., Dodson, R., Orosz, G., Imai, H., \& Frey, S. 2016, AJ, submitted (arXiv:1612.02554)

Rioja, M., Dodson, R., Porcas, R. W., et al. 2009, in 8th Int. e-VLBI Workshop, Geodesy and Astrometry (Trieste: SISSA), 14

Rioja, M. J., Dodson, R., Jung, T., et al. 2014, AJ, 148, 84

Rioja, M. J., Dodson, R., Jung, T., \& Sohn, B. W. 2015, AJ, 150, 202

Rioja, M. J., \& Porcas, R. W. 1998, in ASP Conf. Ser. 144, IAU Coll. 164, Radio Emission from Galactic and Extragalactic Compact Sources, ed. J. A. Zensus, G. B. Taylor, \& J. M. Wrobel (San Francisco, CA: ASP), 95

Ros, E., Marcaide, J. M., Guirado, J. C., \& Pérez-Torres, M. A. 2001, A\&A, 376, 1090

Sokolovsky, K. V., Kovalev, Y. Y., Pushkarev, A. B., Mimica, P., \& Perucho, M. 2011, A\&A, 535, A24

Sovers, O. J., Fanselow, J. L., \& Jacobs, C. S. 1998, RvMP, 70, 1393

Williams, W., Nixon, D., Reilly, H., Withington, J., \& Bathker, D. 1979, DSNPR, 52, 51 